\title{
Ciclo de mejora docente en el Área de Urbanismo y Ordenación del Territorio. Medidas de protección frente a la contaminación visual y perceptiva en el patrimonio urbano
}

DANIEL NaVAs CARRILLO

Departamento de Urbanística y Ordenación del Territorio. Escuela Técnica Superior de Arquitectura. Universidad de Sevilla dnavas@us.es

ORCID: https://orcid.org/0000-0002-9704-3204

D.O.I.: http://dx.doi.org/10.12795/JDU.2018.i01.27

Pp.: 482-499

\section{Resumen}

Esta comunicación aborda el ciclo de mejora implementado en la asignatura "Patrimonio Urbano y Planeamiento" dentro de las actividades programadas en la Fase de Permanencia del Programa de Formación e Innovación docente del profesorado.

El modelo metodológico desarrollado implica un proceso global que incluye la revisión de los contenidos, de las actividades, así como de los procesos de evaluación. Este ha tenido como objetivo situar al 
estudiante en el centro del aprendizaje trabajando en la construcción de su propio conocimiento desde la investigación individual y el trabajo colaborativo.

La mejora se ha centrado en la contaminación visual y perceptiva aplicada a la protección del patrimonio urbano. Se trata de un aspecto que no ha sido abordado en profundidad en el ámbito de la investigación, ni en la práctica profesional.

El desarrollo atestigua, gracias a la exploración inicial y final, que los alumnos han mejorado los resultados en términos globales.

Palabras Clave: patrimonio urbano y planeamiento, grado en fundamentos de arquitectura, docencia universitaria, Experimentación docente universitaria, planeamiento especial de protección.

\section{Contexto de la intervención}

La presente comunicación tiene como objeto aproximarse al ciclo de mejora desarrollado en la metodología docente de la asignatura "Patrimonio Urbano y Planeamiento", optativa que imparte el Departamento de Urbanística y Ordenación del Territorio en quinto curso del Grado en Fundamentos de Arquitectura de la Universidad de Sevilla. La asignatura, que cuenta este curso con 24 alumnos matriculados, de los cuales 16 asisten con regularidad a clase, tiene como objetivo docente familiarizar al estudiante con la figura urbanística del Planeamiento Especial. Entre las competencias específicas con las que se trabaja se debe destacar la aptitud para catalogar el patrimonio y planificar su protección desde una noción amplia del concepto Patrimonio.

Tal como se describe en su programa docente (Universidad de Sevilla, 2018) esta asignatura aborda la figura urbanística del Planeamiento Especial de Protección de forma que al final del cuatrimestre el estudiante sea capaz de elaborar criterios propios y de manejar una metodología que le permita en el futuro abordar profesionalmente la redacción de este tipo de planeamiento. Por este motivo, 
las componentes teóricas y prácticas están estrechamente relacionadas, quedando equilibradas las horas de docencia dedicadas a una y otra. De forma genérica, las cuatro horas de cada clase semanal se organizan de modo que las dos primeras horas se dedican a teoría y las dos siguientes a práctica.

El contenido teórico incorpora conceptos clave sobre la valoración y protección patrimonial, siendo principalmente procedimental, es decir, a través de ejemplos de planeamiento de protección se introducen los principales aspectos a desarrollar en este tipo de trabajo profesional, y que a nivel académico se trabajan en el desarrollo práctico de la asignatura. Se es consciente de que sin formación previa en la temática (no se establece como necesario haber cursado ninguna otra asignatura) la conceptualización teórica de un trabajo de esta índole es difícilmente abordable en un único cuatrimestre.

En cuanto al desarrollo práctico de la asignatura, se tiene en cuenta que la redacción de un plan de estas características es tarea que se realiza en equipo y en un marco temporal que abarca varios años. En este sentido, a partir de la idea de apuntes sobre un Plan Especial (Pérez Cano y Del Espino Hidalgo, 2012), se sigue una metodología de Taller, en la que cada grupo trabaja como si de un equipo redactor de planeamiento especial se tratara, y donde los docentes ejercen de directores en las fases iniciales de este tipo de documento: Información, Diagnóstico y Avance.

\section{Diseño del Ciclo de Mejora}

\section{Principios didácticos}

Tal como se ha descrito anteriormente, frente a la metodología tradicional de transmisión de conocimiento, en la asignatura se aboga por un "aprendizaje crítico natural" 
(Bain, 2007), en el que los estudiantes puedan desarrollar la capacidad de razonamiento autónomo por medio de la resolución de problemas y por tanto fomentando el aprendizaje activo (Santos Asensi y Palacios Vicario, 2017). En ese sentido, diversos autores (Harvey, 1997; Scottish Council Foundation, 2003), mucho antes de que se estuviese abordando en España la adaptación de los planes al Espacio Europeo de Educación Superior, ya hacían hincapié sobre la experiencia práctica como una de las principales herramientas para garantizar la empleabilidad de los titulados.

No se debe olvidar que uno de los objetivos docentes es la capacitación del alumnado en competencia profesionales como potenciales responsables, en este caso, de la protección del patrimonio urbano de nuestras ciudades, aunque es cierto que las técnicas de aprendizaje tradicional en Arquitectura ya se basaban en metodologías similares a lo que hoy conocemos como método de caso, en la que se exige al alumnado tomar decisiones, valorar actuaciones, emitir juicios fundamentados (Servicio de Innovación Educativa de la Universidad Politécnica de Madrid, 2008).

El planteamiento docente de la práctica intenta situar al estudiante en el centro del aprendizaje, fomentando que investigue y trabaje con situaciones nuevas (la redacción de un plan especial); sea creativo, inventando respuestas nuevas y utilizando diversas perspectivas en la resolución del problema (trabaje con patrimonios emergentes y baraje diversos criterios de valoración); desarrolle el pensamiento crítico, no aceptando una única verdad, evaluando diferentes argumentos antes de decidir (trabajando la evolución urbana desde diversas hipótesis evolutivas); se implique y se comprometa de forma activa en la resolución de los problemas, confiando en sus propias capacidades; y trabaje en equipo, resolviendo problemas de forma cooperativa, negociando y consensuando las respuestas (Pérez Cano, Del Espino Hidalgo y Navas Carrillo, 2017).

Tomado este planteamiento docente como punto de partida, el ciclo de mejora busca dar un paso más, trabajando especialmente sobre lo que hasta el momento hemos considerado contenido teórico. Para ello se toma 
como referencia las experiencias que desde el curso 20122013 se vienen desarrollando en el Programa de Formación e Innovación Docente del Profesorado (FIDOP) de la Universidad de Sevilla (Porlán, 2017). Muchas de estas experiencias son deudoras del trabajo de Bain sobre lo que sobre los mejores profesores universitarios hacen (Bain, 2007). Así mismo, cabe destacar como antecedente el ciclo de mejora aplicado a otro contenido de esta misma asignatura el pasado curso 2016-2017 (Navas Carrillo, 2017).

A partir de estos trabajos se adoptan las siguientes ideas, a modo de filosofía docente desde la cual se ha construido la secuencia de actividades del ciclo:

- Realizar una aproximación gradual a los contenidos partiendo de actividades que generen en los estudiantes perplejidad o enigma como estrategia de captación de la atención.

- Situar al estudiante en el centro del aprendizaje trabajando en la construcción colectiva de contenidos mediante procesos de indagación y razonamiento compartido.

- Desplazar la atención sobre el docente, asumiendo un rol de guionista de los contenidos y de la secuencia de actividades y de director de lo que va sucediendo en clase.

- Construir mapas de contenidos, priorizándolos y adaptados a los objetivos - pocos y realistas- fijados en la asignatura y a la secuencia de actividades a realizar.

- Emplear cierta diversidad de actividades, lo que, además de favorecer la adaptación a contenidos y objetivos, conserva el carácter de novedoso frente a los estudiantes.

- Evaluar en diversos momentos (al menos inicial y final) el proceso de aprendizaje de los estudiantes, nuestra actividad como profesor y la metodología empleada.

- Utilizar las actividades de evaluación al inicio de la docencia como herramienta para conocer a los estudiantes y poder adaptar los contenidos a sus necesidades particulares. 
- Adecuar los criterios de evaluación a los objetivos que se han fijado, utilizando las rúbricas como herramienta de trabajo propia pero también como herramienta de consolidación de lo aprendido. En este sentido, se convierte como una actividad más del proceso de aprendizaje.

- Plantear alternativas de aprendizaje que se adecúen a distintos perfiles de estudiantes, fijando compromisos con ellos.

\section{Mapa de contenidos, preguntas-clave $e$ interacciones fundamentales}

Los contenidos desarrollados giran en torno a la protección patrimonial frente a la contaminación visual y perceptiva. La Ley 14/2007, de 26 de noviembre, de Patrimonio Histórico de Andalucía es la primera legislación en todo el territorio nacional en incorporar de forma explícita este concepto. Se trata de un aspecto aún incipiente en la práctica profesional, así como en el ámbito académico. Esta afirmación se sustenta tanto en el número de planes de protección que hayan incorporado medidas específicas al respecto, como en la ausencia de referencias bibliográficas que aborden este aspecto en profundidad.

El objetivo último es que los estudiantes profundicen a través de un ejemplo real, cercano y reconocible por todos, en los diferentes aspectos a tener en cuenta en el análisis y la compresión de este concepto en un espacio patrimonial significativo. Los contenidos se desarrollan pormenorizadamente en el mapa de contenidos recogido en la Figura 1.

\section{Modelo metodológico y secuencia de actividades}

Siguiendo a Finkel (2008), el modelo metodológico desarrollado desplaza al docente del centro del aula dentro de un proceso global que incluye la revisión de los 


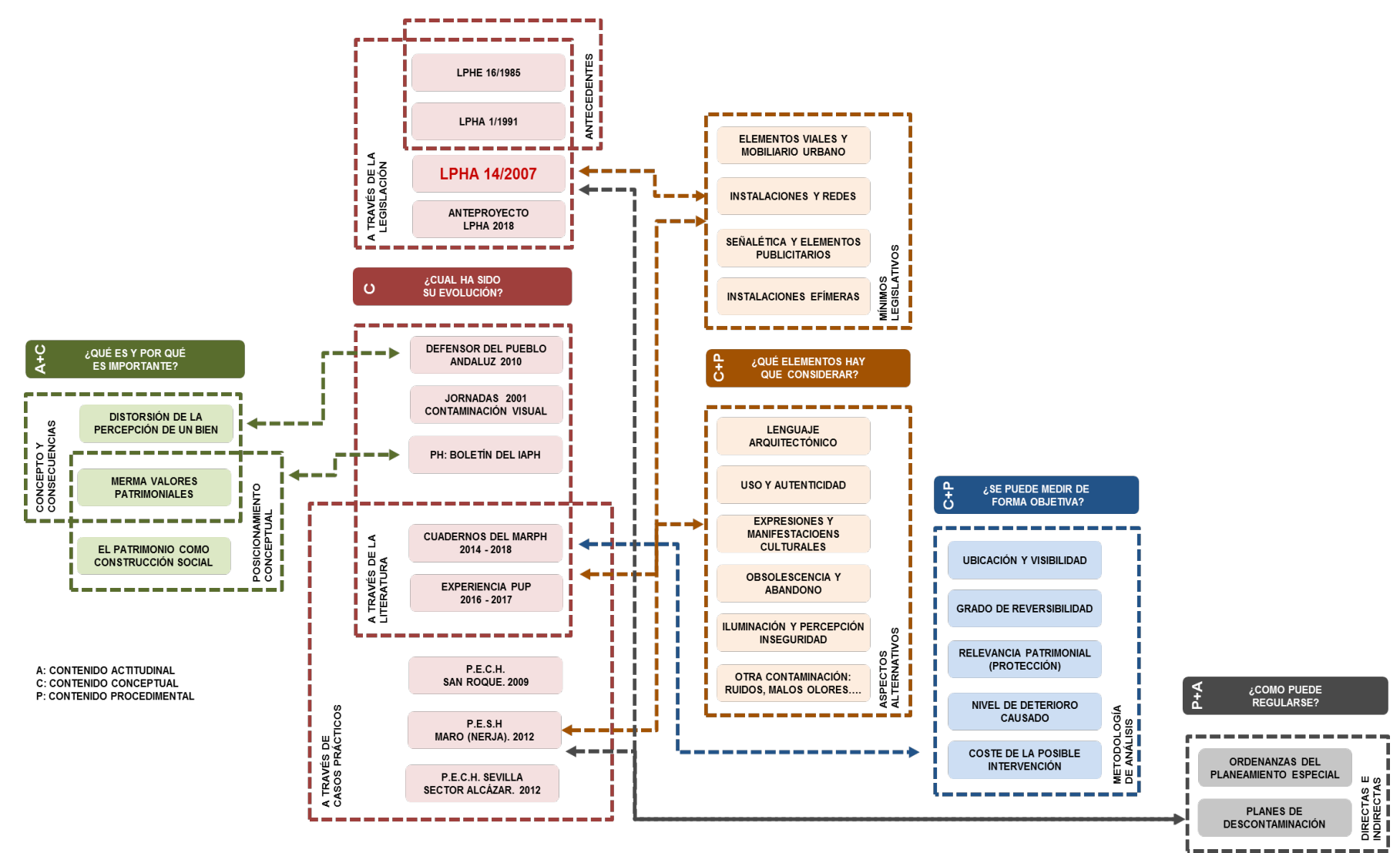

Figura 1. Mapa de contenidos del Ciclo de Mejora. Fuente: Elaboración propia.

Jornadas de Formación e Innovación Docente del Profesorado | № 1 (2018)

(c) E $\Theta$ Esta obra se distribuye con la licencia Creative Commons

cc) (†) $\Theta$ Reconocimiento-NoComercial-SinObraDerivada

Internacional (CC BY-NC-ND 4.0.) 
contenidos, de las actividades, así como de los procesos de evaluación. Se busca que el estudiante se sitúe en el centro del aprendizaje trabajando en la propia construcción del aprendizaje y transformando el modelo actual de clase teórica en procesos que conduzcan desde la investigación individual y el trabajo colaborativo al conocimiento (Herrera, 2017).

El ciclo de mejora ha tratado de repensar la práctica docente desde un enfoque gradual de los contenidos, mediante diversas actividades: Video y lluvia de ideas (30' VD); Evaluación inicial del nivel de conocimientos de los estudiantes (20' - Evi); Análisis de artículos recientemente publicados sobre la temática (40' - Ani); Construcción de una definición conjunta (30' - Dbi). Análisis de metodologías de aplicación (120' - Anii); Presentación de las metodologías de aplicación analizadas (100' - PR); Debate por grupos (30' - Dbii); Debate general (30' - Dbiii); Síntesis del docente (30' - Sdi); Evaluación final del nivel de conocimientos adquirido por los estudiantes (20' - Evii); Reflexión sobre los resultados de la evaluación y síntesis final (30' - Sdii).

Estas actividades construyen el modelo metodológico implementado en el ciclo de mejora. Este queda recogido en la Figura 2, la cual además de distribuir dichas actividades en las dos sesiones desarrolladas evidencia gráficamente el tiempo dedicado a cada una de ellas en el conjunto de la mejora.

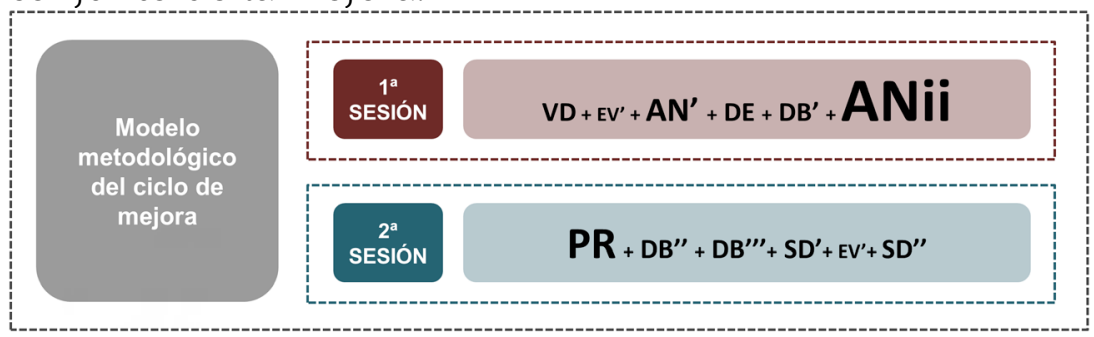

Figura 2. Cuadro resumen del modelo metodológico. Fuente: Elaboración propia. 


\section{Aplicación del Ciclo de Mejora}

\section{Valoración sobre el desarrollo}

El primero de los logros ha estado en implicar a los estudiantes a lo largo del desarrollo de la clase. La primera actividad ha conseguido captar la atención de los estudiantes que, si bien en un primer momento fueron reacios a realizar aportaciones sobre los mismos, tras unos segundos se fueron animando. Buscar la participación de los estudiantes mediante preguntas no es una práctica nueva, pero si el procedimiento. El ir anotando las aportaciones a modo de lluvia de ideas en la pizarra, independientemente de si se trataba de comentarios acertados o no, ayudó a romper el miedo a ser juzgado al que se enfrentan muchos estudiantes. En la práctica habitual, son pocos estudiantes, por no decir ninguno, los que suelen contestar a las preguntas realizadas durante la exposición teórica en clase.

El ciclo ha ayudado a superar las dificultades encontradas en cursos anteriores para que las prácticas abordaran la temática con cierto grado de profundidad. Se les instó a los estudiantes a que una vez asimilada la metodología de análisis que les había tocado estudiar, propusieran algún aspecto de innovación en su aplicación al ámbito de estudio. Sorprendentemente, todos introdujeron de una forma desprejuiciada otras formas de contaminación perceptiva a las clásicas. Significativamente, se abordaron aspectos relativos al acondicionamiento térmico urbano, al valor de uso y autenticidad, a la perspectiva de género en urbanismo, e incluso se relacionó la accesibilidad universal como una de las medidas a considerar en los planes de descontaminación.

La implicación y motivación de los estudiantes ha sido elevada, llevado al extremo que, en la mayoría de los grupos, los estudiantes se han quedado trabajando en clase 
durante la media hora de descanso en la primera de las sesiones. Al final del cuatrimestre los estudiantes de arquitectura suelen encontrarse sobrepasado por la excesiva carga de trabajos prácticos. Pensar en la posibilidad de reducir el trabajo a realizar fuera de clase sin duda ha sido un importante aliciente para ellos, tal como se ha podido testar con algunos de ellos al finalizar la clase.

A pesar de que se había anunciado que se iba a desarrollar una actividad durante la clase con el compromiso de no tener que realizar dicha parte en la entrega final, no asistieron a clase cuatro de los estudiantes. Esto suscitó ciertas discrepancias en algunos de los grupos por lo siguiente. Las actividades del ciclo habían sido diseñadas atendiendo a los grupos ya establecidos para el trabajo práctico se viene realizando durante todo el cuatrimestre. Ahora bien, uno de los grupos se encontró con la ausencia de dos de sus miembros, lo que a priori pensaban iba a dificultar su desarrollo y temían que finalmente no se les eximiera de desarrollar esta parte en la práctica. Se tranquilizó a estos grupos, aclarándoles que la consideración que se iba a realizar era sobre el grado de interés e implicación, y no en el resultado final de la actividad.

Esto se suma a una duda personal en cómo proceder con los estudiantes ausentes, teniendo en cuenta lo fijado por el proyecto docente. Se estimó, y así se transmitió a los estudiantes, que al tratarse de un trabajo en grupo, no se iba a distinguir entre aquellos miembros que habían asistido a clase y aquellos que no, dejando a criterio de cada grupo, como podía afectar esta circunstancia en el desarrollo restante del trabajo práctico. Es una cuestión aún abierta, de la cual habrá que llevar seguimiento hasta final del curso.

Otras de las dificultades encontradas han estado en la capacidad de análisis crítico mostrada por los estudiantes cuando trabajaron en la construcción de una definición propia en base a los textos de investigación. En la mayoría de los casos, se limitaron a componer la definición 
mediante fragmentos literales extraídos del texto. En el trabajo en grupo, no se ha construido una definición conjunta, sino que se ha decidido cuál de todas era más idónea, con matizaciones mínimas. En cualquier caso, este resultado se entiende consecuente al modo de proceder habitual de los estudiantes de grado, y no habiendo sido capaz de transmitir en qué consistía la actividad. Estos aspectos han sido clarificados en la síntesis realizada en base a lo expuesto a los estudiantes, siendo necesario una mayor desarrollo sobre las ideas clave que lo inicialmente previsto.

En relación a la temporización de cada una de las actividades se han tenido que realizar adaptaciones a medida que avanzaba la clase, especialmente en lo que respecta a las presentaciones de los alumnos.

\section{Evaluación del progreso de aprendizaje}

En la Figura 3 se representan los resultados de los test de exploración inicial (T0) y final (T1), con un gradiente de colores de más suave a más fuerte que representan calificaciones en progresión en función de los siguientes criterios de evaluación: La respuesta evidencia asimilación y dominio del contenido general y específico (A); La respuesta evidencia asimilación y dominio del contenido general, pero muestras ciertas carencias en la asimilación de los contenidos específicos (B); La respuesta tiene relación con el nuevo contenido expuesto, pero muestra que no se ha asimilado el nuevo contenido (C); La respuesta no tiene relación alguna con el bloque de contenido expuesto, mostrando que no se ha asimilado el nuevo contenido (D).

En el test inicial se detecta un especial déficit de conocimiento específico sobre la materia, salvo en la cuestión 1, relativa a la definición de la contaminación visual y perceptiva, en la cual el $18 \%$ y $44 \%$ de los estudiantes demostraron respectivamente tener conocimientos específicos y generales. Se trata igualmente de la cuestión que mayor grado de asimilación evidencia el test final, siendo en cualquier caso una pregunta cuya respuesta puede 
construirse en base a los contenidos trabajados con anterioridad en la asignatura.

El contenido trabajado previamente sobre aspectos sobre el entorno de protección de un Bien de Interés Cultural también les ha permitido deducir posibles elementos y factores a considerar en el estudio de la contaminación visual y perceptiva, así como los mecanismos de regulación a través de las ordenanzas. No obstante, las respuestas dadas en la exploración inicial son en la mayoría de casos muy genéricas y, por otra parte, predecibles para estudiantes de quinto curso de arquitectura. Sin embargo, cabe hacer distinción entre ambas cuestiones en la exploración final. En el primero de los casos, el $50 \%$ de las respuestas se han clasificado en el nivel A y el $44 \%$ en nivel B. En el segundo caso, estos porcentajes son menores: 38 y $31 \%$.

Cuestión 1: Definición de la CVyP

$\| A=B=C \equiv D$

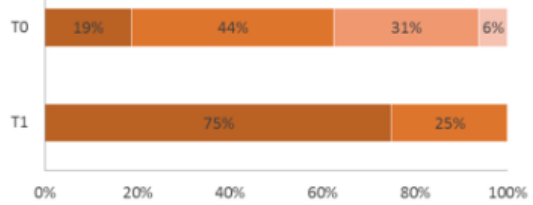

Cuestión 3: Elementos y factores a considerar

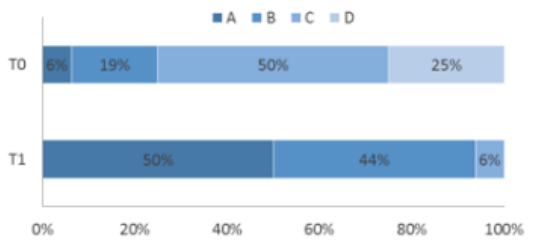

Cuestión 5: Los mecanismos de regulación

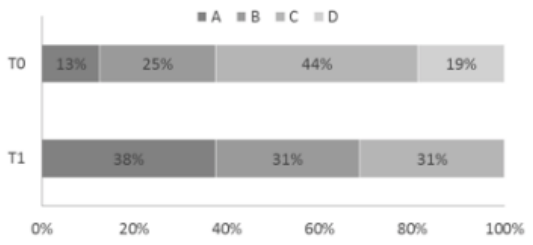

Cuestión 2: Importancia de la CVyP

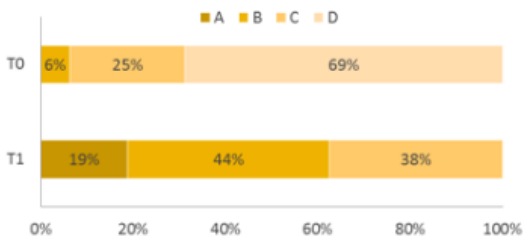

Cuestión 4: Objetividad del análisis

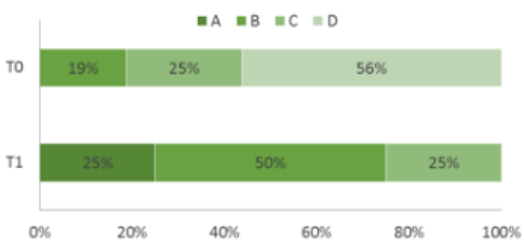

Conjunto de cuestiones

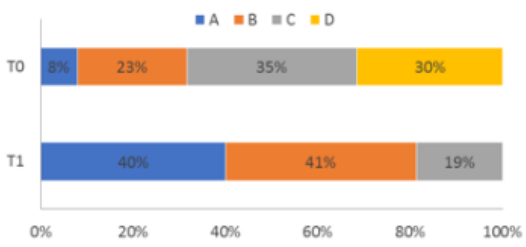

Figura 3. Resultados de las exploraciones inicial y final. Fuente: Elaboración propia.

Jornadas de Formación e Innovación Docente del Profesorado I № 1 (2018) Esta obra se distribuye con la licencia Creative Commons Reconocimiento-NoComercial-SinObraDerivada Internacional (CC BY-NC-ND 4.0.) 
En términos globales los alumnos han mejorado los resultados para el conjunto de respuestas. No obstante, cabe poner el acento sobre los contenidos actitudinales relativo a la importancia de tener en cuenta este contenido en la protección del patrimonio urbano y la necesidad de objetivizar dicho análisis. En ambos casos es detecta un porcentaje elevado de alumnos que no han asimilado los conceptos. En el primer caso solo el 19\% de las respuestas se sitúan en el nivel $A$, mientras que en el segundo este porcentaje aumenta hasta el 25\%. No obstante, es mayoritaria la proporción de estudiantes que se sitúan en el segundo nivel (44 y 50\%) de adquisición de conocimientos. En este sentido, se estima necesario incidir más sobre esta otra dimensión de los contenidos en próximos cursos.

\section{Evaluación del Ciclo de Mejora}

\section{Valoración de la experiencia}

A tenor de los resultados anteriormente recogidos, se ha demostrado la idoneidad del ciclo de mejora para mejorar los resultados del aprendizaje frente a los cursos anteriores. No obstante, se ha diseñado un cuestionario de valoración de la actividad desarrollada y el profesor a través de un formulario de encuestas de goodle, que ha sido enviado a los estudiantes a través del correo electrónico. Esta encuesta ha sido cumplimentada por 15 de los 16 estudiantes que asisten con regularidad a clase y ha versado sobre la actuación general del profesor en el desarrollo del conjunto de actividades (A); el papel que han tenido los/as alumnos/as en el desarrollo de las actividades (B); la utilidad de las actividades realizadas para aprender lo que se pretendía (C); el grado de aprendizaje frente a las clases tradicionales (D); el interés de este modelo frente a las clases tradicionales (E); y la sustitución de las clases tradicionales (teoría-práctica) por otras similares a esta (F). 


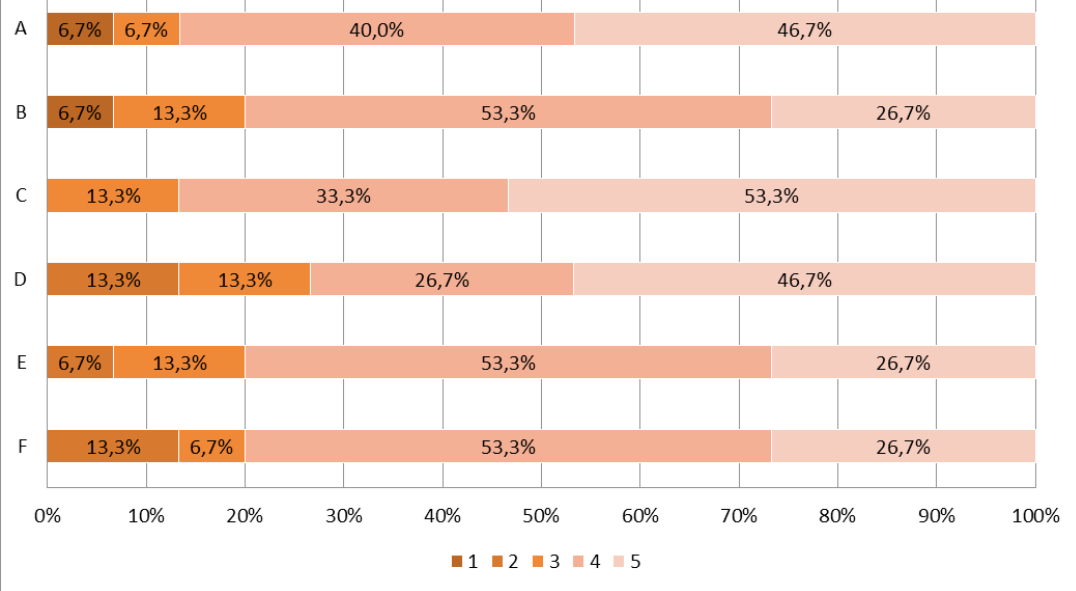

Figura 4. Resultados del cuestionario de evaluación de la experiencia Fuente: Elaboración propia.

Como se observa en la Figura 4 la evaluación de los estudiantes en términos generales es positiva, con algunas discrepancias sobre el papel de los alumnos, especialmente por el aumento de trabajo dentro y fuera del aula. Destacan como positivas el aumento del interés por las clases y la autoevaluación del trabajo de los alumnos. Existen algunas dudas sobre su idoneidad para el aprendizaje, y sobre la idea de que sustituyan a las tradicionales. En cualquier caso, valoración dentro de lo esperado al estar habituados al modelo tradicional. Solo en uno de los casos, se manifiesta total desacuerdo con todos los apartados.

Así mismo este cuestionario permitía comentar cualquier otro aspecto que los estudiantes estimaran relevante sobre la nueva metodología empleada y en general sobre el método de enseñanza empleado en clases. Por ejemplo, que partes le habían gustado más y cuáles menos, o qué actividades complementarías pensaban que también podrían ser útiles en tu aprendizaje. En general, la mayoría de los comentarios destacaban el interés por el modelo implementado, si bien, cabe recoger algunas discrepancias. 
Por una parte, algunos alumnos expresan malestar por el trabajo adicional que les ha supuesto, y que esté no se tuviera en cuenta en la calificación de la asignatura de forma directa. Por otra, también se recogen comentarios que ponen en duda de que esta metodología se pueda aplicar a todos los contenidos de la asignatura.

\section{Conclusiones finales}

El ciclo de mejora se ha diseñado para un contenido específico y acorde al contexto en el que nos encontrábamos. En este sentido, se deben mantener los objetivos que subyacen en las mismas más que las actividades en sí. Tal como se ha mencionado, estos son: hacer trabajar a los alumnos de forma colaborativa, desplazar al docente del centro del aula, convirtiéndose en el guionista y director de lo que ocurre en la clase, y transformar el producto finalista del modelo tradicional de clase teórica o magistral en un proceso que conduzca desde la investigación individual y el trabajo colaborativo al conocimiento.

Se debe aspirar a trabajar fundamentalmente en desarrollar la capacidad de razonamiento y la reflexión crítica entre el alumnado. Se trata de aspectos poco trabajados en el plan docente actual - por inercia de los planes antiguos- y que son fundamentales para la profesión de arquitecto. Se ha señalado como otro de los avances significativos el haber trabajado en la elaboración de mapas de contenidos. En el propio desarrollo de la clase se ha demostrado su utilidad al permitir adaptar las actividades al propio desarrollo de la clase, especialmente en lo que respecta al tiempo empleado en cada una, y el contenido manejado. Por tanto, es uno de los aspectos principales a mantener de forma habitual a la hora de preparar los contenidos de una clase.

También se estiman como fundamentales las actividades vinculadas con la evaluación, tanto del aprendizaje del alumnado mediante exploraciones iniciales y finales, 
como del profesor y del conjunto de actividades desarrolladas. Esto nos permitirá ir continuamente mejorando nuestras clases. Al respecto se estima necesario dedicar algo de tiempo a comentar los resultados de las exploraciones en clase, como actividad de consolidación de lo aprendido o meta-reflexión.

El haber trabajado en un mapa de contenidos previo también es considerado como avance a consolidar en la práctica docente, en tanto que permite organizar el contenido para que el profesor pueda manejarlo mejor. Permite además salir de un modelo encorsetado en el que se suceden los contenidos de forma secuencial, sino que da la posibilidad de construir un modelo más flexible, en el que tener varias entradas a partir del cual ir introduciendo los distintos conceptos que se quieren transmitir. Como en nuestro caso, dota al modelo de capacidad de adaptación ante posibles imprevistos. Pero no sólo se estima útil para el docente, sino que haberlo entregado a los estudiantes nos ha permitido verificar su idoneidad como una herramienta pedagógica más, puesto que les facilita seguir el conjunto de actividades, permitiéndoles comprender de manera global los distintos temas abordados en cada una.

Cabe destacar que el ciclo ha profundizado sobre un aspecto incipiente en materia patrimonial, aún por desarrollar en la práctica profesional, y que sin duda requiere en su enseñanza de un mayor nivel de profundización por su carácter especialmente novedoso. Esto nos lleva a pensar en este recurso como una oportunidad potencial para aumentar el grado de empleabilidad de los futuros egresados, sin duda uno de los principales retos a los que se enfrenta la comunidad universitaria en estos momentos (García Manjón y Pérez López, 2008).

Para concluir se recoge una reflexión personal sobre lo que ha supuesto el curso como docente. Frente a otros compañeros, se cuenta con poca experiencia en docencia, siendo el tercer curso en el que se participa en tareas docentes, las cuales están además limitadas a 60 horas 
por curso. En este sentido, es importante recalcar la importancia que cursos como este tiene para profesores en formación. Las habilidades que se adquieren en este momento serán clave en el futuro (Navas Carrillo, 2017b). Abrir la mirada a otros modelos docentes es una oportunidad para trabajar con ellos desde el inicio de la carrera docente, evitando así tener que redefinirlos más adelante, con las dificultades que conlleva tener que rehusar algo ya interiorizado.

\section{Bibliografia}

Bain, K. (2004). Lo que hacen los mejores profesores universitarios. Valencia: Servei de publicacions. Universitat de València.

Finkel, D. (2008). Dar clase con la boca cerrada. Valencia: Servei de publicacions. Universitat de València.

García Manjón, J.V. y Pérez López, M.C. (2008). Espacio Europeo de Educación Superior, competencias profesionales y empleabilidad. Revista Iberoamericana de Educación, 46(9), 1-12.

Harvey, L. (1997). Graduates'Work: Organisational Change and Students'Attributes. Birmingham: University of Central England, Centre for Research Quality.

Fernando Herrera, R. (2017). Aprendizaje basado en proyectos colaborativos de entornos de programación a partir de proyectos de ingeniería civil. Revista Electrónica Educare, $21(2), 1-18$

Navas Carrillo, D. (2017a). "Mejoras en la metodología docente de la asignatura Patrimonio Urbano y Planeamiento". En: IV Jornadas de Formación e Innovación Docente. Sevilla: Universidad de Sevilla.

Navas Carrillo, D. (2017b). "La formación inicial del profesorado como oportunidad para la innovación y mejora de las metodologías docentes". En Peña Acuña, B. (Coord.) Contribuyendo a una nueva docencia a partir del EEES. Madrid: Tecnos.

Pérez Cano, M.T. y Del Espino Hidalgo, B. (2012). La protección del patrimonio edificado a través del planeamiento: la figura del Plan Especial y su aplicación a la protección del patrimonio de ciudades de tamaño pequeño y medio. Colección: Contenidos Plan Propio de Docencia. Sevilla: Repositorio de Objetos de Aprendizaje de la Universidad de Sevilla. 
Pérez Cano, M.T., Del Espino Hidalgo, B. y Navas Carrillo, D. (2017). Patrimonio Urbano y Planeamiento: La protección del Paisaje Patrimonial a través del Planeamiento Especial. En García Bujalance, S. Territorio, Turismo y Paisaje: metodologías docentes. Málaga: Editorial Geometría.

Porlán, R. (2017). Enseñanza Universitaria. Como mejorarla. Universidad de Sevilla. España: Ediciones Morata.

Santos Asensi, C. y Palacios Vicario, B. (2017) "Metodologías activas en el espacio superior de enseñanza". En Peña Acuña, B. (Coord.) Contribuyendo a una nueva docencia a partir del EEES. Madrid: Tecnos.

Scottish Council Foundation (2003). Higher Education: Higher Ambitions? Edimburgo: Critical Thinking.

Servicio de Innovación Educativa de la Universidad Politécnica de Madrid (2008). El Método del Caso. Guías Rápidas para nuevas metodologías. Madrid.

Universidad de Sevilla (2018). Programa de la asignatura Patrimonio Urbano y Planeamiento. Grado en Fundamentos de Arquitectura. Departamento de Urbanística y Ordenación del Territorio. E.T.S. de Arquitectura. Curso 2017-18. Recuperado de http://www.us.es/estudios/grados/plan 233/asignatura_2330069\#programa (9 de junio de 2018).

Jornadas de Formación e Innovación Docente del Profesorado | № 1 (2018) Esta obra se distribuye con la licencia Creative Commons 